\title{
Boosting reliability over AWGN networks with average power constraints and noiseless feedback
}

\author{
Anant Sahai, Stark C. Draper, and Michael Gastpar \\ Department of EECS, University of California, Berkeley, CA, 94720 \\ \{sahai, sdraper, gastpar\}@eecs.berkeley.edu
}

\begin{abstract}
For the point-to-point additive white Gaussian noise (AWGN) channel with noiseless feedback and an average power constraint, Schalkwijk and Kailath's scheme achieves a doublyexponential decay of the probability of error. While some coding schemes for networks with noiseless feedback incorporate variations on the Schalkwijk-Kailath scheme, they do not in general achieve better than single-exponential decays in their probabilities of error everywhere in their achievable rate regions. We give a technique that can boost the reliability as high as desired of any from a large class of block coding schemes for networks with feedback. The technique relies crucially on the average nature of the power constraints. We explain and illustrate our results in the context of Ozarow's feedback strategy for the AWGN multiple-access channel.
\end{abstract}

\section{INTRODUCTION}

For the point-to-point AWGN channel with an average power constraint, Shannon [1] shows that feedback does not increase capacity. That noiseless feedback of the channel output is nevertheless useful for AWGN channels is shown, e.g., by Schalkwijk and Kailath [2], where they construct a simple code that achieves a doubly-exponential decay of the error probability with increasing block-length. Later, Kramer [8] analyzed a different model in which such noiseless output feedback is replaced by noiseless decision feedback, i.e., exact knowledge of what decision the decoder is about to make. Even with this weaker model, Kramer is able to show faster than doubly-exponential decay of the error probability.

For AWGN networks, the situation is different in two respects. First, feedback does increase the achievable rates in many cases. Second, network reliability functions (error exponents) without feedback are mostly unknown. Indeed, many existing coding theorems for AWGN networks with feedback and average power constraints do not yield spectacular error exponents. Whenever typicality arguments are used, the dependence of the error probability on the block-length $N$ becomes obscured. While there is usually an exponential term that comes from the difference of the rate and the relevant mutual information expression, there is also an $\epsilon$ term that covers the probability of the atypical set. Given the nature of the AWGN channel, in most cases we can apply a Chernoff bound and see that $\epsilon$ is also an exponentially small function of the block length. However, the exponent involved can be quite low.

The situation is best illustrated by the AWGN multipleaccess channel (MAC) with noiseless output feedback. Ozarow determines the full capacity region in [5], as illus-

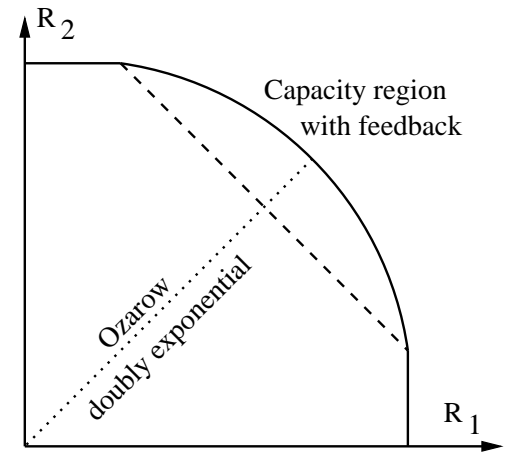

Fig. 1. Schematic rendering of the capacity region of the AWGN MAC without feedback (dashed line) and with feedback (solid line). Ozarow's achievability proof leads to doubly exponential error probability decay for symmetric rate points (dotted line), but only to singly exponential error probability decay for all other rate points.

trated in Figure 1. Without feedback, the capacity region has the pentagonal shape indicated by the dashed line. With feedback, this region can be extended to the curved shape given by the solid line in the figure. To establish the curved region, Ozarow specifies a coding scheme inspired by SchalkwijkKailath. This scheme achieves any symmetric rate pair, those indicated in the figure by the dotted line. Furthermore, the error probability decays doubly-exponentially for these rate pairs. To attain the remainder of the capacity region, Ozarow superimposes a regular feedback-free block code. Hence, for any non-symmetric rate point, the overall error probability only decays singly exponentially — the errors of the superimposed block code dominate. Our investigations are motivated by the question of whether this effect is fundamental, or an artifact of Ozarow's specific scheme.

In this paper, we present a novel scheme that achieves a reliability better than any single exponential everywhere in the interior of Ozarow's capacity region. The strategy is related to Kramer's technique [8]. In addition to the MAC, we show how to apply the technique to attain similarly high reliabilities for many other AWGN networks.

\section{BASIC IDEA}

The approach we take builds on a key insight for using feedback to improve reliability in the context of average cost constraints: "speak quietly, but carry a growing stick." In other words, over time save up power so that when the channel 
behaves atypically, you can respond by blasting much more power than you do on average. Strategies of this kind were proposed for point-to-point channels by Kramer [8], and more recently in [10], [12].

In this paper we expand upon this insight, specifying a general framework for super-reliable communication networks with AWGN links, feedback, and average power constraints. Feedback is used so that information sources can detect decoding errors and initiate retransmissions. The idea of retransmission in case of detected error is old, and has also been extensively explored in the context of discrete memoryless channels. In particular, if we only constrain the average block length, rather than fixing a set block length, then the availability of feedback makes much higher reliabilities possible. Both Burnashev [3] and Yamamoto-Itoh [4] give schemes that attain the maximum possible reliability in this context. Further, both of their schemes operate with sourceinitiated retransmissions, as oppose to sink-demanded, as in the case of Forney [6]. In contrast to the variable-length nature of these schemes, we use a fixed block length. ${ }^{1}$ And, instead of exploiting a weaker average-block-length constraint, we are able to increase reliability by exploiting an average power constraint. Note that Wyner shows in [13] that the SchalkwijkKailath strategy only achieves singly-exponential error decay when a peak power constraint in imposed. In further contrast to these earlier papers, we apply our ideas to networks.

We term the resulting increase in decoding reliability a "boost" because our strategy can be used in conjunction with many baseline block-oriented strategies. The baseline strategy need only satisfy a few characteristics, discussed further in Section IV. The boosting technique can even be applied to achievability strategies not yet invented. Indeed, this is one of the main morals of the paper - in AWGN networks operating under average power constraints and having sufficiently capable feedback, it is not really necessary to be concerned with block-coding error exponents. We can always improve reliability through boosting.

The outline of the paper is as follows. The boosting technique is introduced and analyzed in the context of Ozarow's scheme in Section III. In Section IV we discuss the applicability of the scheme more widely, and sketch the strategy for general networks. A number of network examples of the application of the technique is given in Section V. Comments and conclusions are given in Sections VI-VII.

\section{BOOSTING OZAROW'S RELIABILITY THROUGH EMERGENCY RETRANSMISSIONS}

In this section we develop our scheme as applied to the specific context of boosting the reliability of Ozarow's strategy. In the multiple-access network Ozarow considers [5] there are two transmitters $A$ and $B$ with average power constraints $P_{A}$ and $P_{B}$, and with independent messages, $m_{A}$ and $m_{B}$, of rates $R_{A}$ and $R_{B}$, respectively. The channel is a memoryless AWGN channel with output $Y_{i}=X_{A, i}+X_{B, i}+Z_{i}$ where

\footnotetext{
${ }^{1}$ It is straightforward to generalize our ideas to the variable-length context.
}

$X_{A, i}$ and $X_{B, i}$ are the channel inputs of transmitters $A$ and $B$, respectively, and $Z$ is zero-mean AWGN with variance $\sigma^{2}$. The channel inputs are functions of the messages and previous channel outputs as $X_{A, i}=f_{A}\left(m_{A}, Y_{1}, \ldots, Y_{i-1}\right)$ and $X_{B, i}=f_{B}\left(m_{B}, Y_{1}, \ldots, Y_{i-1}\right)$.

Our strategy starts by picking a rate pair $\vec{R}=\left[\begin{array}{ll}R_{A} & R_{B}\end{array}\right]$ in the interior of Ozarow's achievable region. We boost its reliability as follows:

Setup: Pick a positive scalar $\beta<1$ so that the rate vector $(1 / \beta) \vec{R}$ is still achievable. This is possible since $\vec{R}$ is assumed to be in the interior of the rate-region. The constant $\beta$ provides the "slack" needed to accommodate boosting. If $\vec{R}$ is close to the boundary of the achievable rate region, then $\beta$ is close to one.

Given a sufficiently long block-length $N$, divide it into two phases: a data transmission phase of length $\beta N$, and an alarm and retransmission phase of length $(1-\beta) N$.

Data Transmission: For the first $\beta N$ channel uses ${ }^{2}$, use the Ozarow scheme operating at rate $(1 / \beta) \vec{R}$. Because of the output feedback, at the end of this phase the transmitters know whether the receiver has correctly decoded their messages.

Alarms and Retransmissions: The remaining $(1-\beta) N$ channel uses are split between the two transmitters. For simplicity we assume that they are divided equally. The interval $[\beta N+1, \beta N+0.5(1-\beta) N]$ is dedicated to transmitter $A$, and $[\beta N+0.5(1-\beta) N+1, N]$ to transmitter $B$.

If no errors are detected at the end of the transmission phase, both transmitters sit idle during this phase. If any errors are detected, the system goes into emergency mode. In the first of its channel uses, the relevant transmitter raises an alarm by sending a "very loud" signal. After sending its alarm, the transmitter follows up with a retransmission of its message. Because of the time-division, retransmissions experience no interference. During retransmission the required data of $N R_{A}$ (respectively, $N R_{B}$ ) is transmitted at rate roughly $R_{A} / 0.5(1-$ $\beta$ ) (respectively, $R_{B} / 0.5(1-\beta)$ ). If the overall scheme is operating close to the outer bound of the achievable rate region, then $\beta \simeq 1$, and the retransmission rates are very large. To be able to support such high retransmission rates at high reliability we need to use a very large retransmission power. As we show, because retransmissions are quite rare, we can support very high power retransmissions without exceeding the system's average power constraints. Further note that while the point-to-point Schalkwijk-Kailath scheme could be used for retransmissions, we show that standard block codes suffice.

Meanwhile, the receiver listens for alarms at times $\beta N+1$ and $\beta N+0.5(1-\beta) N+1$. If it detects an alarm in the former, it interprets that as a NAK. It then tries anew to decode transmitter $A$ 's message from its observations in the interval $[\beta N+2, \beta N+0.5(1-\beta) N]$. If it detects an alarm at the latter time, it does the same for transmitter $B$ 's message.

\footnotetext{
${ }^{2}$ For notational simplicity we treat $\beta N$, and similar quantities later, as integers. They should be rounded to the nearest integers for correctness.
} 


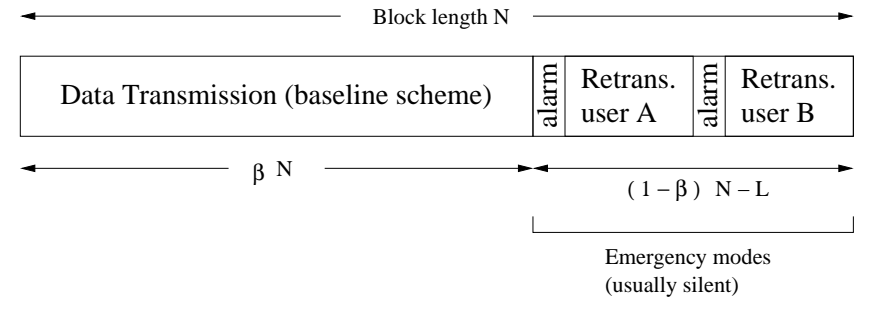

Fig. 2. Division of channel uses into the phases of boosting scheme: data transmission using baseline scheme and alarms and retransmissions. Because the data transmission phase is most often successful, most transmitters are usually silent during emergency mode, thereby bringing down the average energy usage.

\section{A. Average power usage}

The key to the strategy is that emergency mode is activated only very rarely. During most alarm and retransmission phases transmitters remain silent. They can therefore transmit at very high powers during emergencies, and still meet their average power constraints.

Since whether or not emergency mode is activated depends on whether data transmission was successful, the energy used by the scheme in any particular block is random. Let us assume that all block-error probabilities in the data transmission phase are going to zero with block-length at least as fast as

$$
\operatorname{Pr}[\text { error }] \leq N^{-\Delta},
$$

for some $\Delta>0$. Ozarow's scheme satisfies (1) as its probability of error decays to zero exponentially fast in $N$.

Under the assumption (1) we bound the expected energy used per block. This will tell us the total emergency energy $\vec{E}_{e}=\left[E_{A} E_{B}\right]$ available for alarms and retransmissions.

$$
N \vec{P}=\beta N \vec{P}+\operatorname{Pr}[\text { error }] \vec{E}_{e} \leq \beta N \vec{P}+N^{-\Delta} \vec{E}_{e},
$$

where the average power constraint $\vec{P}=\left[P_{A} P_{B}\right]$. The $\beta N \vec{P}$ term comes from the energy used every block during the data transmission phase, and the $N^{-\Delta} \vec{E}_{e}$ term from the emergency phase contribution. The inequality is term-by-term. Using (1) in (2) and solving for $\vec{E}_{e}$ gives

$$
\vec{E}_{e}=\left[\begin{array}{c}
E_{A} \\
E_{B}
\end{array}\right] \geq N^{1+\Delta}(1-\beta)\left[\begin{array}{l}
P_{A} \\
P_{B}
\end{array}\right]=N^{1+\Delta}(1-\beta) \vec{P} .
$$

The important observation here is that the emergency mode transmitter energies grow on a per-transmitter basis faster than the block-length $N$.

\section{B. Raising the alarm}

Alarms are raised by sending a large power pulse at the appropriate time. If we transmit an alarm with minimum power $P_{\min }$, and use a basic maximum-likelihood detector, then the probability of mis-detecting either alarm signal is upper bounded (via the union bound) by

$$
\operatorname{Pr}[\text { alarm error }] \leq 2 \exp \left\{-P_{\min } / 8 \sigma^{2}\right\} .
$$

By using one-half the total emergency energy for each alarm, we get $P_{\min }=0.5 \min \left\{E_{A}, E_{B}\right\} \geq 0.5 N^{1+\Delta}(1-$ $\beta) \min \left\{P_{A}, P_{B}\right\}$. Using this in (4) shows that the probability of an alarm error decreases to zero faster than any singleexponential in $N .^{3}$ Further, if the error probability in (1) actually goes to zero exponentially fast - as in Ozarow's scheme - then $\vec{E}_{e}$ in (3) grows exponentially fast in $N$, and the alarm error (4) will decay at a doubly-exponential rate.

\section{Error as a function of block length}

We now analyze the overall system error probability as a function of block length. Because we use a time-division strategy during the alarm and retransmission phase, when active each link is a simple interference-free point-to-point AWGN channel. For example, the information stream from transmitter $A$ is retransmitted at rate $R_{A} / 0.5(1-\beta)$. In order to communicate this rate reliably, it must be below the link capacity, i.e.,

$$
\frac{R_{A}}{0.5(1-\beta)} \leq \frac{1}{2} \ln \left[1+\frac{\check{P}_{A}}{\sigma^{2}}\right],
$$

where $\check{P}_{A}$ is the average power available for retransmission over the link. This power comes from the $0.5 E_{a}$ left over after we have already spent half the energy on the alarms. Each retransmission takes roughly $0.5(1-\beta) N$ channel uses. This means that user $A$ 's retransmission power is

$$
\check{P}_{A}=\frac{0.5 E_{A}}{0.5(1-\beta) N} \geq P_{A} N^{\Delta} .
$$

The important thing to notice about (6) is that it is growing with $N$. A similar result holds for user $B$.

Using (6) in the right-hand side of (5) shows that the capacity of the channel during retransmission grows like $0.5 \Delta \ln [N]$. However, the communication rate required during retransmission, i.e., the left-hand side of (5) is constant in $N$. Since this is an AWGN channel, the random coding error exponent $E_{r}\left(r, \check{P}_{A} / \sigma^{2}\right)$ at all fixed rates $r>0$ goes to infinity as $\check{P}_{A} / \sigma^{2}$ increases. For $\check{P}_{A}$ large we get $E_{r}\left(r, \check{P}_{A} / \sigma^{2}\right) \geq$ $\left.0.5 \ln \left[0.5\left(1+\check{P}_{A} / \sigma^{2}\right)\right]\right)-r$ (see [7], p. 340). This means that the error probability of user $A$ 's retransmission is bounded by

$$
K \exp \left\{-\frac{(1-\beta) N}{2}\left[\frac{1}{2} \ln \left(\frac{\sigma^{2}+P_{A} N^{\Delta}}{2 \sigma^{2}}\right)-\frac{2 R_{A}}{(1-\beta)}\right]\right\}
$$

where $(1-\beta) N / 2$ is the block-length used during retransmission. The error probability in (7) decays exponentially in $N \ln (N)$ - faster than any single-exponential in $N$. If the error probability in (1) were to go to zero exponentially fast then, since the retransmission power in (6) would be exponential in $N$, the retransmission error probability would go to zero exponentially fast in $N^{2}$.

The total probability of error on the block is bounded by the sum of the error probabilities on the alarms plus the error probabilities on the emergency retransmissions. Since

\footnotetext{
${ }^{3}$ Note that in many networks the alarms can be eliminated by merging them with the retransmissions. We can use Gallager's random code construction for the AWGN channel to guarantee that all codewords have a high average power. If we add a special all-zeros codeword to the codebook to communicate the lack of an alarm, then alarm detection can be accomplished by looking at the total received energy during the retransmission phase.
} 
all of these error probabilities decay faster than any singleexponential, and there are a finite number of them, we achieve an overall probability of system error that decays faster than any single-exponential in the block length.

\section{Discussion}

There are four things to note about the boosting strategy that underly its applicability to other networks. First, the only type of feedback required is decision feedback, not output feedback. Although, of course, whether or not one has output feedback may effect the achievable rate region. Second, during boosting we can use a standard feedback-free block code. This means that the retransmission phase is not tied into any kind of feedback-dependent coding strategy. Third, the error decay required on the data transmission block (1), is quite slow, and thus can be satisfied by a wide range of strategies. Finally, the division of the emergency energy to alarms and retransmissions is done evenly, without any particular regard to transmit powers or receiver signal-to-noise ratios. One could optimize these choices, but such optimization is not needed to show faster-than-exponential decay in the error probabilities.

\section{General Network STRATEGY}

In this section we discuss the general characteristics that a system must have for our strategy to be applicable. We then quickly summarize the strategy in general networks as an extension of that described for the MAC problem.

The first assumption has to do with the class of networks. The network must consist of channels that are constrained to operate under average cost constraints. Thus, during temporary "emergencies" - when the channel has behaved atypically poorly - the peak cost can greatly exceed the average constraint. This allows us to deliver data quickly and at high reliability.

The second assumption has to do with the feedback channel. We assume there is some implicit or explicit instantaneous "tentative decision" feedback from every information sink to its corresponding information source. By this, we mean that at the conclusion of the data transmission phase, each source knows what decision the corresponding sink would make, if the sink were forced to decide right then. E.g., the noiseless output feedback in Ozarow's MAC setting has this property.

Third, we assume the existence of some baseline blockcoding scheme. The baseline scheme determines the achievable data rates of the overall strategy, while boosting determines its reliability. The major requirement is that the target rate point must lie strictly within the achievable region for the baseline scheme. The boosting strategy we propose cannot be used to improve the reliability of a baseline scheme that operates right on the boundary of a closed achievable region.

The main result of this paper is that for any network that satisfies the above three conditions, the reliability of the boosted baseline strategy can be made better than any singly exponential function of the block length.
Sketch of general strategy: Say we have a network with $S$ nodes, and also a baseline strategy that supports any achievable rate vector $\vec{R}$ at an error probability satisfying (1).

To implement our strategy, as before, we pick a $\beta<1$ such that $(1 / \beta) \vec{R}$ is still achievable. We divide the $(1-\beta) N$ channel uses of emergency mode into $L$ time-slots. Since the network consists of $S$ nodes, the maximum number of information source-sink pairs is $S^{2}$. Further, the network may have a multi-hop relay character if some of the source-sink pairs have no direct path. As it takes at most $S-1$ hops for a message to arrive at its corresponding sink, an upper bound on the number of time slots needed is $L=S^{3}$. During each slot a single transmitter-receiver pair is active, the rest of the network is silent. The retransmission rates are therefore upper-bounded by $S^{3} \vec{R} /(1-\beta)$. Since $S$ is assumed to be finite, this is a vector of finite rates.

The bound on the expected emergency energy equals (3), as before, and again we spend half the energy on the alarms and half on the retransmissions. The alarm probability is again given by (4), except for two substitutions. First, the constant 2 out front is replaced by $S^{3}$, again from the union bound. Second, now the ratio $P_{\min } / \sigma^{2}$ is replaced by the minimum ratio, across all the transmitter/receiver pairs, of the receiver signal-to-noise ratio during alarm transmissions. This ratio still grows faster than $N$, so we get a faster-than-exponential decay. A similar argument also holds for the retransmission probability of (5). Since all these probabilities of error decay faster than any single exponential, and there are a finite number of links (bounded by $S^{3}$ ), the overall probability of error decays this fast as well.

\section{NETWORK EXAMPLES}

In this section we apply the boosting strategy to a number of elementary networks. In addition, we show an example the two-way channel - that does not satisfy the conditions of Section IV, and for which boosting does not work.

Multiple access channel: For the MAC problem with a single receiver, we set $L=S-1$ which gives each source the chance to raise an alarm.

Broadcast channel: For the broadcast channel with a single transmitter and many receivers, we set $L=S-1$. As before all receivers monitor their alarm time. Note that we do not care if the broadcast channel is physically degraded, stochastically degraded, or non-degraded.

Single-layer relay channel: Consider a relay channel with a single source-destination pair, and a layer of $S-2$ helper relays in between. These relays can hear the source and help get the message to the destination. We assume that the source can be heard at the destination, although perhaps in an attenuated manner, and that it has access to noiseless feedback from the destination. In this case we can pick $L=1$ since there is a direct path from source to sink. Had there not been a direct path $L>1$ would be required. Similarly, the direct path from source to sink suffices for the emergency topology used during retransmission. The relays stay silent. 
Interference channel: In the simple interference channel, there are $S / 2$ transmitters and $S / 2$ receivers, all of which face a direct path and various cross-channel interferences. We require noiseless feedback from each sink to its corresponding source. We need $L=S / 2$ time slots to raise the alarm since each transmitter requires its own alarm. With this choice, each source-destination retransmission occurs in the absence of interference. Strategies for interference networks with feedback that generalize Ozarow's approach are developed in [9].

Two-way channel: The two-way channel is an illustration of a network where boosting does not work. In the standard Gaussian two-way channel, both parties observe the same output $Y=X_{A}+X_{B}+Z$, and want to transmit at rates $R_{A}$ and $R_{B}$ to each other. Since both transmitters see a common output, the sources effectively have noiseless output feedback. But this noiseless feedback in not sufficient for each to determine whether its sink has decoded correctly. However, if we add explicit decision feedback, that would trivialize the problem. If such explicit feedback links were present, one might as well send the original messages along them!

\section{Comments And Challenges}

The critical assumption underlying our approach is the average nature of the power constraint. With a peak-power constraint, there would be a limit on our retransmit power, and so we could not boost reliability everywhere in the interior of the achievable region while maintaining a fixed block length.

Even with average power constraints, the cases of noisy or finite-rate noiseless feedback are challenging because error detection capability would then not be perfect. Further, we really need to take account of the time required to feedback the decisions from the decoder before an encoder can raise its alarm. The pipelining techniques of [11] might be address the latter, but it is unclear how finite-rate feedback would impact the result. We suspect that constrained feedback will impose an upper limit on how high we can boost reliability.

We have applied boosting to the MAC, broadcast, singlelayer relay, and interference problems. In all those cases, the feedback is effectively link-by-link. For network with larger depths, having noiseless feedback from all downstream nodes can be a very strong assumption. For such networks, a link-bylink instantiation of this boosting scheme will not work unless the strategy is either a decode-and-forward strategy or at least one that can be conceptualized in terms of reliable links. In non decode-and-forward relaying strategies, the presence of link-by-link feedback may not allow intermediate nodes to detect that an error has occurred and react appropriately.

A similar approach can also be used to boost block-coding reliability in the context of fast fading. If the power is high enough, the probability of block error using traditional schemes is often limited by the exponentially small probability of outage when the fading realization is atypically bad. Boosting can be used whenever the transmitter can realize that it is in outage, and boost its transmit power accordingly. The lesson is that the transmitter should water-pour over the fading states only up to a point. When the fast fading realization is so bad that it threatens to put the entire block into outage, the transmitter needs to increase transmit power to fight it.

\section{CONCLUSION}

For AWGN problems with noiseless feedback from downstream nodes, there is no need to bother trying to calculate block-coding reliability functions. The result here shows that we can boost the reliability to better than any exponential providing that the baseline probability of error satisfies (1).

In fact, the technique described here can be applied recursively to boost the reliability yet again. If, for instance, the error probability in (1) decayed exponentially fast in the block length, than every additional boost would add to the exponent: a single boost gives exponential decay in $N^{2}$, a double boost in $N^{3}$, and so on. Even greater boosts are possible. For example, if we can run Schalkwijk-Kailath during retransmissions, we would get a doubly-exponential decay. Alternately, we could use Kramer-like strategies for retransmission.

Stepping back, a main interpretation of this result seems to be an indictment of the model. A pure average power constraint in conjunction with noiseless feedback is too lax a restriction on the system model.

\section{ACKNOWLEDGMENT}

This work was supported in part by the NSF Grant No. CNS-0326503.

\section{REFERENCES}

[1] C. E. Shannon, "The zero error capacity of a noisy channel," IRE Trans. Inform. Theory, vol. IT-2, pp. 8-19, Sep. 1956.

[2] J. P. M. Schalkwijk and T. Kailath, "A coding scheme for additive noise channels with feedback-I: No bandwidth constraint," IEEE Trans. Inform. Theory, vol. IT-12, pp. 172-182, Apr. 1966.

[3] M. V. Burnashev, "Data transmission over a discrete channel with feedback, random transmission time." Problemy Peredachii Informatsii, 12(4):10-30, Oct.-Dec. 1976.

[4] H. Yamamoto and K. Itoh, "Asymptotic performance of a modified Schalkwijk-Barron scheme for channels with noiseless feedback," IEEE Trans. Inform. Theory, vol. IT-25, pp. 729-733, Nov. 1979.

[5] L. H. Ozarow, "The capacity of the white Gaussian multiple access channel with feedback," IEEE Trans. Inform. Theory, vol. IT-30, pp. 623-629, Jul. 1984.

[6] G. D. Forney, "Exponential error bounds for erasure, list, and decision feedback schemes," IEEE Trans. Inform. Theory, Vol 14, pp 206-220, Mar. 1968.

[7] R. G. Gallager, Information Theory and Reliable Communication, John Wiley and Sons, 1968.

[8] A. J. Kramer, "Improving communication reliability by use of an intermittent feedback channel," IEEE Trans. Inform. Theory, vol. IT15 , pp. 52 - 60, Jan. 1969.

[9] G. Kramer, "Feedback strategies for white Gaussian interference networks," IEEE Trans. Inform. Theory, vol. IT-48, pp. 1423-1438, Jun. 2002.

[10] A. Sahai and Q. Xu, "The anytime reliability of the AWGN + erasure channel with feedback," Allerton Conf. on Commun., Control, and Comp., Monticello, IL, Sep. 2004.

[11] A. Sahai and T. Simsek, "On the variable-delay reliability function of discrete memoryless channels with access to noisy feedback," IEEE Workshop on Inform. Theory, San Antonio, TX, Nov. 2004

[12] A. Sahai, S. Avestimehr, and P. Minero, "Anytime communication over the Gilbert-Eliot channel with noiseless feedback," Accepted to ISIT 2005

[13] A. D. Wyner, "On the Schalkwijk-Kailath coding scheme with a peak energy constraint," IEEE Trans. Inform. Theory, vol. IT-14, pp. 129 134, Jan. 1968. 